\title{
Research of Medical Monitoring System Based on WSN
}

\author{
Zhifang Wang ${ }^{*}$ and Peng Liu
}

Polytechnic College of Hebei University of Science and Technology, Shijiazhuang, Hebei, 050018, China

\begin{abstract}
A medical monitoring system based on wireless sensor network (WSN) is proposed in this paper. It designs cluster - tree wireless sensor network nodes, which consist of an acquisition module, a microprocessor unit and wireless communication module. The design makes it possible that the data is transmitted accurately from acquisition nodes to sink nodes through the router nodes, and the collection and transmission of wireless sensor network nodes are accurate and stable. It can greatly expand the capacity of monitor, improve the monitoring management, and increase monitoring efficiency.
\end{abstract}

Keywords: Medical monitoring, S3c2440, WSN, Zigbee.

\section{INTRODUCTION}

At present, most medical monitoring systems in the hospital are connected to a wired network. The sensor probes are used to connect the patients with the monitoring devices for signal transmission. The doctors can make the medical diagnosis and disease analysis according to the data and waveform shown in the displays. However, the wired monitoring equipments have many disadvantages, such as high price, huge volume, large demand [1,2], and inconvenience for patients. So the wireless system is necessary in the future.

The application of the wireless sensor network (WSN) in medical monitoring systems can give more free movement to the patients by reducing the wire between the medical sensors and monitoring equipments, and obtain more accurate data from the target monitors. It can greatly expand the capacity of monitor, improve the monitoring management, and increase monitoring efficiency. At present, the medical monitoring systems using GPRS, Bluetooth, WiFi and other wireless technology have been reported at home and abroad. Although these equipments could meet some specific requirements, they have many problems, such as high cost, complex protocol and large power consumption. This paper proposed a medical monitoring system based on WSN, which is based on ZigBee. This system has low power consumption, low cost, large network capacity, and it not only solves the problem of poor mobility and high cost of traditional health care system, but also improves the scalability of the network [3, 4].

\section{ARCHITECTURE OF WSN}

ZigBee standard supports three network topology structures, star network, cluster-tree network and mesh network. The structure of star network is simple with low energy consumption, but is generally used for short distance

\footnotetext{
*Address correspondence to this author at the Hebei University of Science and Technology, No. 70, Yuhua Road, Shijiazhuang 050018, China; Tel: +86-15531037361; E-mail: wzfhbkd@163.com
}

transmission. The structure of mesh network has high robustness with high energy consumption, while the structure of cluster-tree has low energy consumption and simplicity as the structure of star network, and large extended range as mesh network. Because medical monitoring systems need a wide range, but the sensing range of nodes is limited, the structure of cluster-tree network is chosen in the medical monitoring system. The architecture of the sensor network system is shown in Fig. (1).

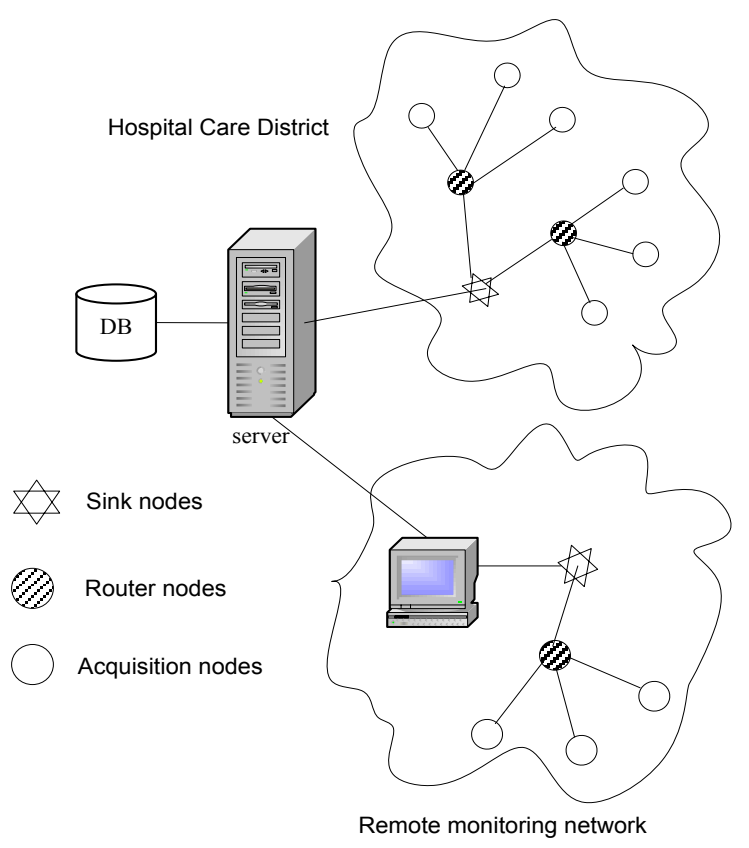

Fig. (1). Network structure diagram.

The acquisition nodes are placed on patients' beds or the consulting rooms to do the collection and data preprocessing on human physiological parameters, and then send them to sink nodes by router nodes. The sink nodes could open and maintain the entire network. They receive data sent by the network nodes, and communicate with the 
monitoring computer to achieve the analysis and real-time monitoring to a variety of physiological parameters of patients [5].

The system can also monitor the chronic patients at home or in community. The collected data are sent to the medical monitoring center on the Internet, so that doctors can observe the data and provide necessary consulting services.

\section{HARDWARE DESIGN OF WSN NODES}

A. Hardware design of acquisition nodes and router nodes. To transmit and receive the data, acquisition nodes and router nodes have the same hardware design, which consists of a power module, a sensor module, a microprocessor unit, a ZigBee wireless communication module, a LCD display module and a keyboard, while they just have different software design. The structures of acquisition nodes and router nodes are shown in Fig. (2).

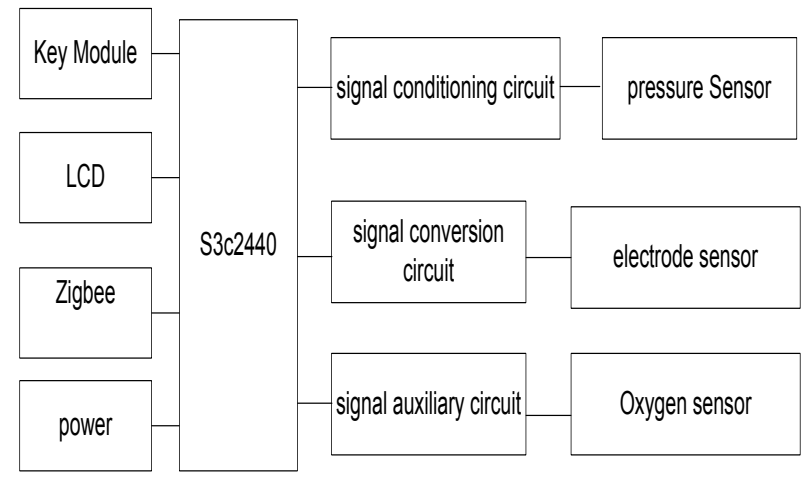

Fig. (2). The structure of acquisition nodes and routing nodes.

Microprocessor unit: In the design, the nodes of microprocessor unit use low-power ARM9 microprocessor S3C2440, which has two UART interfaces, two SPI interfaces with buffer and variable data length. One UART interface is used to connect the ZigBee wireless communication module. The sensors transform the human physiological parameters into the voltage and current signals to the signal conditioning circuit. After being sorted out, the signals are sent to the ADC interface of S3C2440 to do the A/D sampling. Then S3C2440 does the processing and feature extraction and sends to Zigbee wireless communication module CC2530 through the SPI interface [6].

Zigbee wireless communication module: Because of the characteristics of electromagnetic radiation, electromagnetic wave of the monitoring equipments in the hospital cannot interfere with others. Thus, the wireless medical monitoring equipments must solve this problem. ZigBee technology is a wireless transmission technology with low speed and low energy consumption. It is based on the IEEE802.15.4 standard [7], and its working frequency is $2.4 \mathrm{GHz}$. In the design, the CC2530 chip module following IEEE 802.15.4 standard has data modulation and demodulation [8] with bidirectional transceiver. It is shown in Fig. (2). The information is transmitted from the interface SPI of the S3C2440 processor to CC2530, and modulated by spread spectrum O-QPSK and sent out through the pathway of antenna. RF signals received by the antenna are transmitted to CC2530. The original data after demodulation and de-spread is transmitted to the
S3C2440 through the interface SPI. S3C2440 controls the pathway of transceiver [9-13].

Acquisition module of sensor: The medical monitoring systems mainly carry on the test to blood pressure, the electrocardiogram (ECG), the pulse and other physiological parameters. The piezoresistive pressure sensors are used to test blood pressure and the pulse [14], and the electrode sensors are used to monitor the electrocardiogram signal.

Signal acquisition by piezoresistive pressure sensors: The piezoresistive pressure sensors can obtain blood pressure and pulse signals, which are usually weak at the level of $\mathrm{mV}$. While the input voltage in $\mathrm{A} / \mathrm{D}$ converter of the microprocessor S3C2440 is $0 \mathrm{~V}$ to $3.3 \mathrm{~V}$. So signals should be amplified and filtered by the signal conditioning circuit (as shown in Fig. 2) to suit the A/D converter.

Signal acquisition by electrode sensor: The ECG signals are obtained by electrodes which are in contact with human body, and known as lead. The electrode sensors draw in human standard lead signal by the front electrode. Because of the high impedance (about $100 \mathrm{~K})$, weak voltage $(0.05 \mathrm{mV} \sim$ $4 \mathrm{mV}$ ), and high surrounding electromagnetic interference (especially the $50 \mathrm{~Hz}$ ), the ECG signals do the amplification and interference elimination by ECG signal conversion circuit (as shown in Fig. 2). First, weak ECG signals are amplified through the pre-amplifier circuit, and become low common-mode signals with low temperature drift and low noise. Direct circuit signals and low frequency interference signals are removed by high-pass filter circuit, and $50 \mathrm{~Hz}$ frequency interference is further filtered by notch filter. Finally, the useful ECG signals are obtained by the main amplifier and the low frequency filter circuit.

Signal acquisition by oxygen sensor: Oxygen measurement principle uses two specific wavelengths to achieve the measurement of pulse oximetry. The two wavelengths are $660 \mathrm{~nm}$ and $940 \mathrm{~nm}$. The signal auxiliary circuit (shown as Fig. 2) obtains a certain extent signals after amplification, filtering. The signals are sent to the A/D conversion circuit, which converts analog signal into digital signal. Then, S3C2440 calculates the oxygen value of saturation.

The power module makes $5 \mathrm{~V}$ direct circuit go through AMS1117-3.3 and AMS1117-1.8 respectively and their voltages are transformed into $3.3 \mathrm{~V}$ and $1.8 \mathrm{~V}$, which supply power for chip peripherals and kernel on the board. Display module uses LCD12864 to show current pulse, blood pressure, oxygen saturation and other physiological parameters. Keyboard module is mainly used to set the alarm value (about blood pressure, pulse, blood oxygen saturation and etc.).

B. The hardware design of sink nodes: The sink nodes are mainly used to communicate with the host computer. The hardware structure is shown in Fig. (3). The nodes consist of microprocessor module S3C2440 and wireless communication module CC2530, LCD display module, RS232 serial interface module and power module.

\section{SOFTWARE DESIGN OF WSN NODES}

A. Software design of acquisition nodes: The network system does not support data inquiries from the sink nodes to 


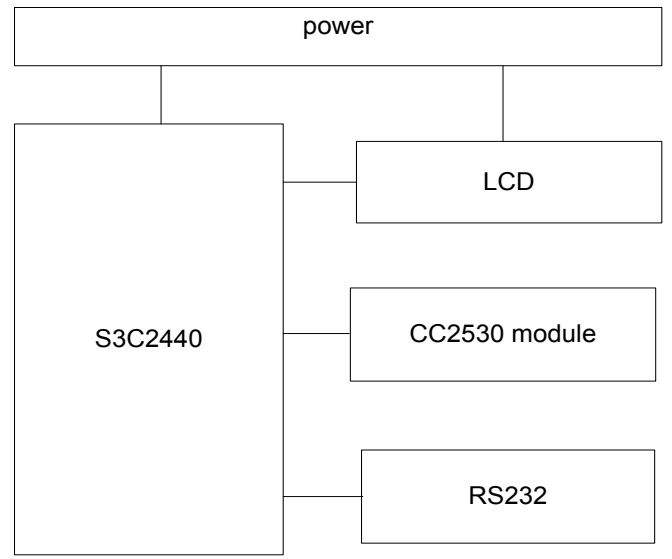

Fig. (3). The structure of sink nodes.

the router nodes and the acquisition nodes, but the data transmission from the acquisition nodes and router nodes to the sink nodes $[15,16]$. When the physiological parameters collected by the acquisition node are higher than the threshold, the acquisition nodes would send data packets of physiological parameters and alarm information packages to the router nodes. The data packets go through the sink nodes to the monitoring computer timely to warn doctors to take corresponding measures. In order to reduce the power consumption of data communication and network, the software is designed to send data packets when the detected data exceeds the preset threshold. It is shown in Fig. (4a).

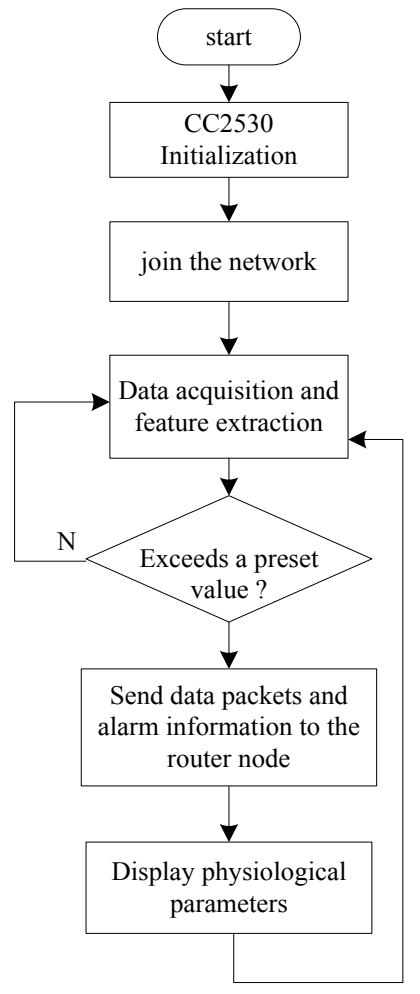

(a) Acquisition nodes

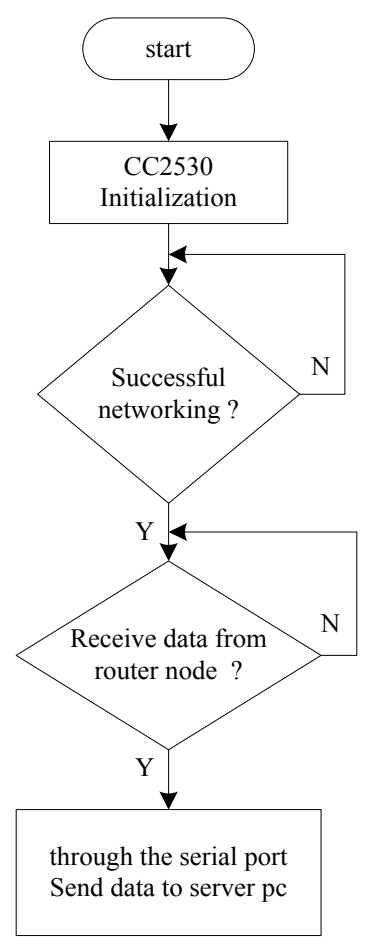

(b) Sink nodes
Fig. (4). Software flow chart of acquisition nodes and sink nodes.

B. Software design of router nodes: The router nodes are in charge of transforming the data packets. When receiving a data packet, they would check its destination address. If the destination address is correct, this data packet would be sent to the adjacent router nodes or sink nodes after being solved by the network layer. If not, this data packet would be discarded. In order to reduce power consumption, the router nodes would go into hibernation if no packet is received [17].

C. Software design of sink nodes: When the program of sink nodes starts, CPU, chip CC2530 and SPI interface are initialized, and a network is built by the function. After the successful establishment of the network, the acquisition nodes and the router nodes are allowed to join in. After that, the sink nodes would process the data packets from the router nodes, and upload them to PC in the monitor center through the serial port. The software design is shown in Fig. (4b).

\section{EXPERIMENT RESULTS AND ANALYSIS}

In order to test the connectivity and accuracy of wireless sensor network, a cluster - tree wireless sensor network is built. The structure of network topology is shown in Fig. (5).
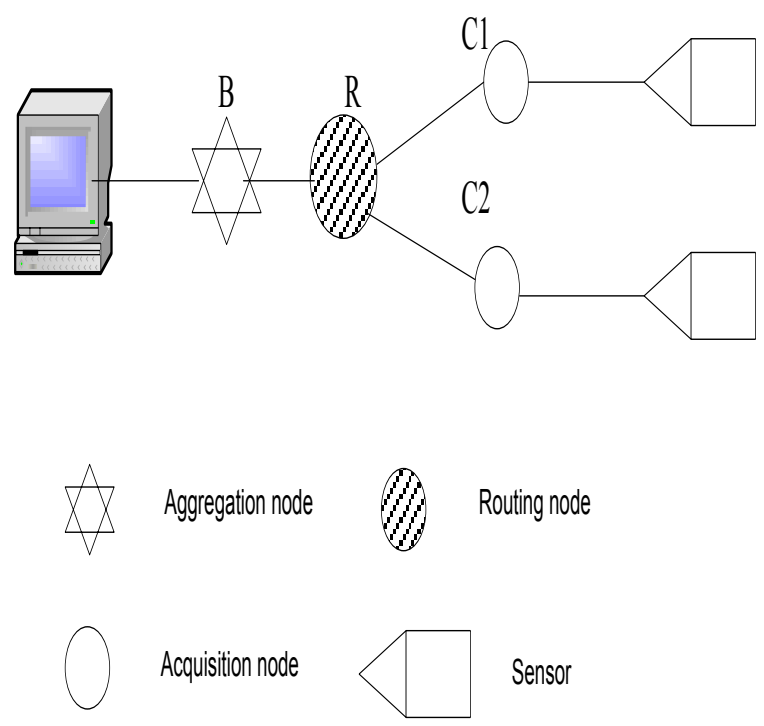

Fig. (5). The structure of network topology.

In the Experiment, the pressure sensor BP3000T is used, Its zero drift is $\pm 30 \mathrm{mV}$, its output voltage is $100 \mathrm{mV} \pm 30$ $\mathrm{mV}$, and its pressure is $40 \mathrm{kPa}$. The pressure sensor is connected to the acquisition nodes $\mathrm{C} 1$ and $\mathrm{C} 2$, and the data collected by $\mathrm{C} 1$ and $\mathrm{C} 2$ is transmitted to the router node $\mathrm{R}$. The sink node B transmits the data through RS232 to the monitoring computer [18]. The blood pressure and pulse are tested in the experiment. The amplitude coefficient of noninvasive blood pressure measurement is adopted to test systolic blood pressure and diastolic blood pressure. $A(S P) / A(M P)=\lambda_{1} \quad$ (systolic pressure coefficient), $A(D P) / A(M P)=\lambda_{2} \quad$ (diastolic pressure coefficient), $A(M P)$ is the maximum value of blood pressure amplitude, $\lambda_{1}$ is between $0.3 \sim 0.75, \lambda_{2}$ is between $0.45 \sim 0.90$. The pulse number $n=60 * N 1 * f / N$, ( $n$ is pulse rate (unit: time/ 
Table 1. Experimental data.

\begin{tabular}{|c|c|c|c|c|c|c|c|c|c|c|}
\hline \multirow{2}{*}{$\begin{array}{c}\text { Measure } \\
\text { Time }\end{array}$} & \multirow{2}{*}{ Teste } & \multicolumn{3}{|c|}{$\begin{array}{c}\text { Blood pressure and Pulse Sensor } \\
\text { Measurement }\end{array}$} & \multicolumn{3}{|c|}{$\begin{array}{c}\text { Mercury sphygmomanometer } \\
\text { Measurement }\end{array}$} & \multicolumn{3}{|c|}{ Error/kPa } \\
\hline & & $\begin{array}{l}\text { Pulse Rate } \\
\text { (bout/min) }\end{array}$ & $\begin{array}{l}\text { SBP } \\
(\mathrm{kPa})\end{array}$ & $\begin{array}{l}\text { DBP } \\
(\mathbf{k P a})\end{array}$ & $\begin{array}{l}\text { Pulse rate } \\
\text { (bout } / \mathrm{min} \text { ) }\end{array}$ & $\begin{array}{l}\text { SBP } \\
(\mathrm{kPa})\end{array}$ & $\begin{array}{l}\text { DBP } \\
(\mathbf{k P a})\end{array}$ & $\begin{array}{l}\text { Pulse rate } \\
\text { (bout } / \mathrm{min} \text { ) }\end{array}$ & $\begin{array}{l}\text { SBP } \\
(\mathbf{k P a})\end{array}$ & $\begin{array}{l}\text { DBP } \\
(\mathrm{kPa})\end{array}$ \\
\hline Morning & Jim & 90 & 13.7 & 9.02 & 89 & 13.3 & 8.39 & +1 & +3 & +4 \\
\hline noon & Jim & 96 & 14.1 & 9.09 & 99 & 14.9 & 9.51 & -4 & -4 & -3 \\
\hline afternoon & $\mathrm{Jim}$ & 93 & 16.5 & 8.68 & 98 & 16.0 & 8.22 & -3 & +3 & +4 \\
\hline Morning & Ali & 69 & 17.5 & 11.31 & 65 & 17.9 & 10.28 & +4 & -2 & +3 \\
\hline noon & Ali & 78 & 18.5 & 10.89 & 78 & 17.6 & 11.31 & +2 & +4 & -3 \\
\hline afternoon & Ali & 86 & 16.2 & 9.94 & 80 & 17.1 & 10.38 & +5 & -2 & -4 \\
\hline
\end{tabular}

Table 2. Network data transmission test structure.

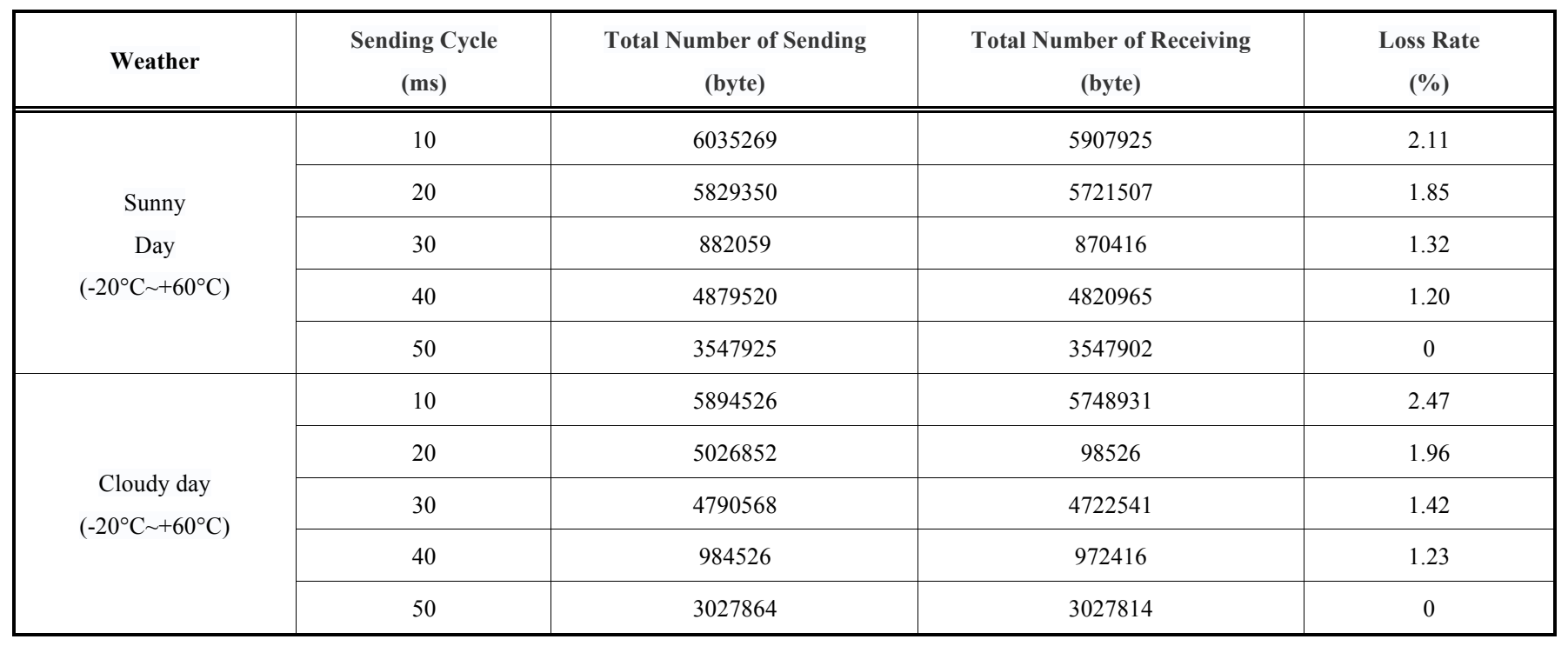

min), $\mathrm{N}$ is sampling frequency, $\mathrm{N} 1$ is sampled pulse number and $f$ is the sampling frequency). Since the pressure sensor has zero drift, it needs to be calibrated. The sensor is connected to mercury sphygmomanometer by cuff, and its readings and the voltage values in discharging process are recorded when the tests are in the normal physiological state. The review averaged 20 test values, and then made the linear approximation by least square method. The function between cuff pressure and voltage value is: $\mathrm{F}=1.1 \mathrm{~V}-0.02$, ( $\mathrm{F}$ is the pressure $(\mathrm{kPa})$, and $\mathrm{V}$ is amplified voltage by $\mathrm{AD})$. It is shown in Table 1. The blood pressures in the table were tested by mercury sphygmomanometer and by sensor nodes at the same time. From Table 1, it shows that the accuracy of blood pressure measurement can reach $0.53 \mathrm{kPa}$, and the data acquisition of sensors [20] has high accuracy.

In the process of testing, $10 \mathrm{~ms}, 20 \mathrm{~ms}, 30 \mathrm{~ms}, 40 \mathrm{~ms}$ and $50 \mathrm{~ms}$ were used to test under different weather conditions. The loss rate of packets was 0 when the period of transmission is $50 \mathrm{~ms}$. In order to record the sending and receiving of packets, serial port debugging program is used [21-22]. The test results are shown in Table 2 . From Table 2 , by compar- ing the measured data the record, it could be concluded that data transmission by wireless sensor nodes in the laboratory is more reliable and accurate.

\section{CONCLUSION}

In this paper, a medical monitoring system based on wireless sensor network (WSN) is designed. Serious sensors are adopted to collect the ECG, blood pressure, pulse and blood oxygen saturation, and constitute a cluster-tree network. In this paper, it is realized that the data is transmitted accurately from acquisition nodes to sink nodes through the router nodes. The WSN nodes technology based on ARM and ZigBee makes medical monitoring management convenient and telemedicine possible, and improves the traditional medical monitoring system further.

\section{CONFLICT OF INTEREST}

The authors confirm that this article content has no conflicts of interest. 


\section{ACKNOWLEDGEMENTS}

Declared none.

\section{REFERENCES}

[1] Z.B. Liang, and P. Li, "Design and implementation of wireless sensor networks gateway based on ZigBee technology", Internet of Things Technologies, vol. 22, pp. 133-137, 2013.

[2] Y.Y. Liu, L. Zhu, and D.C. Huang, "The System Design of Farmland Environmental Monitoring Based on GPRS and Wireless Sensor Network", Journal of Agricultural Mechanization Research, vol. 35, pp. 229-232, 2013.

[3] T.H. Qin, and D. Xiaoqian, "Application of ZigBee technology in w ireless sensor network", Instrumentation Technology, vol. 36, pp. 57-59, 2012.

[4] R.F. Wan, and C.b. Xiu, "The design of wind speed measurement system based on ZigBee technology", Journal of Central South University (Science and Technology), vol. 23, pp. S1: 162-165, 2013.

[5] Z. Tian, "ARM9 Embedded Experimental Development Practice", Beijing: Beihang University Press, 2010.

[6] P.H. Zou, and Y. Liu, "A data-aggregation scheme for WSN based on optimal weight allocation", Journal of Networks, vol. 9, pp. 100-107, 2014.

[7] Z.B. Zhu, G. Wang, and D. Zhang, "A design of mine WSN layout strategy", Procedia Engineering, vol. 15, pp. 5508-5513, 2011.

[8] P.M. Pawar, R.H. Nielsen, N.R. Prasad, and R. Prasad, " Mobility impact on cluster based MAC layer protocols in wireless sensor networks", Wireless Personal Communications vol. 74, no. 4, pp. 1213-1229, 2014.

[9] M. Pandey, and S. Verma, "Privacy provisioning in wireless sensor networks", Wireless Personal Communications vol. 75, no. 2, pp. 1115-1140, 2014.

[10] Y. C. Kan, Hsueh-CL., and W. Sung, "Developing an intelligent control system of automatic window motor with diverse wireless sensor network devices", Sadhana, vol. 39, no. 4, pp. 809-818, 2014.

[11] M.J. Chae, H.S. Yoo, J.Y. Kim, and M.Y. Cho, "Development of a wireless sensor network system for suspension bridge health monitoring", Automation in Construction, vol. 21, pp. 237-252,2011.
[12] L. Jiping, L. Shouyin, and W. Shixun, "A design of remote computer house monitoring and control system based on ZigBee WSN", EN, vol. 4, no. 12, pp. 233-240, 2012.

[13] S.K. Noh, K.S. Kim, Y.K. Ji, and T.H. Kim, "Design of a room monitoring system for wireless sensor networks", International Journal of Distributed Sensor Networks, vol. 2013, p. 7, 2013.

[14] E. Köppe, and M. Bartholmai, "Wireless sensor network with temperature compensated measuring technology for long-term structural health monitoring of buildings and infrastructures", Rocedia Engineering, vol. 25, pp. 1032-1036, 2012.

[15] M. Víctor, P. Semp, and C. Santonja, "Integrated sensor and management system for urban waste water networks and prevention of critical situations", Computers Environment and Urban Systems, vol. 36, no. 1, pp. 65-80, 2011 .

[16] M.B. Yassein, and M. Hassan, "Improvement on the lifetime of the WSN using energy efficiency saving of leach protocol (new improved LEACH)", Sensors \& Transducers, vol. 130, no. 7, pp. 142$153,2011$.

[17] D. Chen, Z.X. Liu, L.Z. Wang, J.Y. Chen, and H. Li, "Natural disaster monitoring with wireless sensor networks: a case study of data-intensive applications upon low-cost scalable systems", Mobile Networks and Applications, vol. 18, no. 5, pp. 651-663, 2013.

[18] Y.C. Zhong, L. Cheng, L. Zhang, Hamid Reza Karimi, P. GarciaTeodoro, and Z. Sun, "Energy-efficient routing control algorithm in large-scale WSN for water environment monitoring with application to three gorges reservoir area", The Scientific World Journal, vol. 2014, p. 9, 2014.

[19] K. Daesik, K. Yusik, S. Seokil, and C. Ken, "Real time traceability and monitoring system for agricultural products based on wireless sensor network", International Journal of Distributed Sensor Networks, vol. 2014, p. 7, 2014.

[20] K.Y. Lian, S. Hsiao, and W.T. Sung, "Intelligent multi-sensor control system based on innovative technology integration via ZigBee and Wi-Fi networks", Journal of Network and Computer Applications, vol. 36, no. 2, pp. 756-767, 2013.

[21] E. Cortez Nicolás, V. Filho Jozué, G. Baptista Fabricio, "Ensign and implementation of wireless sensor networks for impedancebased structural health monitoring using ZigBee and Global System for Mobile Communications", Journal of Intelligent Material Systems and Structures, vol. 26, no. 10, pp. 1207-1218, 2015.

[22] H.Y. Zhu, P.B. Wu, Z. Jing, and W.X. Teng, "Environment monitoring system research based on the ZigBee wireless sensor network technology", Sensors \& Transducers, vol. 170, no. 5, pp. 94100, 2014. 